\title{
Linx
}

Revue des linguistes de l'université Paris X Nanterre

$54 \mid 2006$

La cause : approche pluridisciplinaire

\section{De la concession à la cause, et de la cause à la condition}

Denis Le Pesant

\section{(2) OpenEdition}

\section{Journals}

Édition électronique

URL : http://journals.openedition.org/linx/503

DOI : $10.4000 /$ linx.503

ISSN : 2118-9692

Éditeur

Presses universitaires de Paris Nanterre

\section{Édition imprimée}

Date de publication : 1 juin 2006

Pagination : 61-71

ISSN : 0246-8743

\section{Référence électronique}

Denis Le Pesant, «De la concession à la cause, et de la cause à la condition », Linx [En ligne],

54 | 2006, mis en ligne le 01 août 2007, consulté le 30 avril 2019. URL : http://

journals.openedition.org/linx/503 ; DOI : 10.4000/linx.503

Département de Sciences du langage, Université Paris Ouest 


\title{
De la concession à la cause, et de la cause à la condition
}

\author{
Denis Le Pesant, Université Lille 3 \\ MoDyCo (UMR 7114)
}

Il est bien connu que la conjonction causale parce que autorise la permutation de ses arguments, la grammaticalité et l'interprétabilité étant conservées ${ }^{1}$. Les deux phrases résultant de l'opération ne sont évidemment pas synonymes : par exemple, du point de vue pragmatique, l'une est une assertion portant sur l'existence d'un fait, l'autre est une assertion abductive (ceci pouvant être décrit aussi en terme de différence de portée du connecteur, cf. infra Section 2.4) :

(1a) Assertion sur l'existence d'un fait :

Cette eau bout parce qu'elle a été portée à au moins $100^{\circ} \mathrm{C}$.

(1b) Abduction :

Cette eau a été portée à an moins $100^{\circ} \mathrm{C}$, parce que (= puisque) elle bout $=$ "Si je dis que cette eau a été portée à au moins $100^{\circ} \mathrm{C}$, c'est parce qu'elle bout».

\footnotetext{
1 A la suite de Z. Harris 1976, nous analysons les connecteurs, quelle que soit la partie du discours à laquelle ils appartiennent, comme des prédicats à deux places prenant pour arguments soit des propositions, comme dans les exemples ci-dessous, soit des syntagmes nominaux à tête prédicative. Nous allons évoquer principalement dans cet article les conjonctions si, parce que, puisque, bien que, quoique, malgré que, ainsi que les adverbes pourtant, cependant et néanmoins. Nous aurions pu aussi compléter l'analyse en introduisant les connecteurs prépositionnels à condition de, à cause de, malgré, en dépit de, etc. Enfin, il sera fait allusion dans la Section 2.3 aux connecteurs causatifs verbaux (causer, permettre, empêcher etc.).
} 
D'autre part, nous montrerons dans la Section 2.2 que la négation externe, appliquée à (1a) produit (2a), et appliquée à (1b) produit (2b) :

(2a) Cette eau ne bout pas, bien qu'elle ait été portée à au moins $100^{\circ} \mathrm{C}$.

(2b) Cette eau n'a pas été portée à au moins $100^{\circ} \mathrm{C}$, bien qu'elle bouille.

Au sein des phrases à connecteur de concession (2a) et (2b), le phénomène d'inversion parfaite de l'ordre des arguments qui prévalait dans le couple (1a) et (1b) ne se manifeste pas : la négation a changé de place. Est-ce à dire que les arguments de bien que ne peuvent pas permuter, la grammaticalité et l'interprétabilité étant conservées ? Il n'en est rien, comme le montrent les exemples (3a) et (3b) :

(2a) Cette eau ne bout pas, bien qu'elle ait été portée à au moins $100^{\circ} \mathrm{C}$.

(3a) Cette eau a été portée à au moins $100^{\circ}$, bien qu'elle ne bouille pas.

(2b) Cette eau n'a pas été portée à au moins $100^{\circ} \mathrm{C}$, bien qu'elle bouille.

(3b) Cette eau bout, bien qu'elle n'ait pas été portée à au moins $100^{\circ} \mathrm{C}$.

Tout cela suggère que la permutation des arguments de bien que est d'une nature complètement différente de celle des arguments de parce que.

A quelle opération syntactico-sémantique doit-on relier le phénomène de permutation des arguments du connecteur concessif ? C'est un des problèmes que nous voudrions résoudre. Pour ce faire, nous allons recourir à la notion logique de «contraposition», en l'étendant à la description syntactico-sémantique et en en élargissant le champ d'application (cf. Section 4). Selon cette analyse, (3a) est la contraposée de (2a) et réciproquement ; (3b) est la contraposée de (2b) et réciproquement (cf. Section 4.3). La contraposition jouant un rôle important dans la syntaxe et la sémantique d'autres connecteurs que concessifs, nous allons définir, autour de cette notion, un ensemble de régularités linguistiques liant les éléments de toute une classe de structures concessives, causales et conditionnelles (cf. Section 5). Comme ces régularités sont de nature non transformationnelle, nous aurons à nous expliquer sur le choix de décrire, en syntaxe et en sémantique, des phénomènes que beaucoup de linguistes rapportent à la logique ou à la structure du monde (cf. Section 7).

\section{La permutation des arguments des connecteurs de concession logique}

La permutation des arguments de certains connecteurs concessifs est commentée dans plusieurs grammaires traditionnelles, ainsi que par M.-A. Morel (1996:53), qui écrit :

Pourtant et cependant se différencient des autres marqueurs par plusieurs propriétés : ils peuvent relier deux propositions qui sont permutables entre elles, sans que le sens de la relation concessive entre les deux propositions soit modifié. R.-L. Wagner qualifiait 
les marqueurs ayant cette propriété de marqueurs 'porte-manteau'. La valeur de la relation marquée par ces deux adverbes est celle de la concession logique ${ }^{2}$.

M.-A. Morel continue avec deux exemples :

Notre voiture a 100.000 bornes, elle a pourtant (cependant) très bien roulé

Notre voiture a très bien roulé, elle a pourtant (cependant) 100.000 bornes.

A propos de l'adverbe pourtant, elle précise :

Ayant une valeur proche de celle de la conjonction bien que, il s'en différencie toutefois en ce que la proposition dans laquelle il s'insère peut, selon les cas, être interprétée comme la principale, ou comme la subordonnée.

Selon nous, la propriété « porte-manteau » est aussi bien vérifiée avec les conjonctions qu'avec les adverbes concessifs. Pour s'en convaincre, il suffit de considérer la vingtaine d'exemples de phrases à conjonction bien que, quoique et malgré que que donne Sandfeld (1936:370-373 (\$S 223-224)) : elles admettent presque toutes avec naturel la permutation des arguments. Voici les deux premiers et les deux derniers exemples de concession proposées par Sandfeld dans ce passage :

(4a) Il n'a pas cessé son travail, bien qu'il soit malade.

Il a tout mangé, bien qu'il n'eût pas faim.

Malgré que sa voix füt grelottante et cassée, il savait encore bien la faire entendre.

Elle avait une figure espiègle d'enfant, bien qu'elle approchât de la trentième année.

Permutons les arguments. Nous obtenons :

(4b) Bien qu'il n'ait pas cessé son travail, il est malade.

Bien qu'il ait tout mangé, il n'avait pas faim.

Malgré qu'il sût encore bien faire entendre sa voix, elle était grelottante et cassée Bien qu'elle eût une figure espiègle d'enfant, elle approchait de la trentième année

Nous reviendrons sur cette question à la Section 4.3.

\section{De la concession à la causalité}

\subsection{L'énoncé à connecteur de concession logique comme expression de la négation externe d'un énoncé à connecteur de causalité}

M.-A. Morel (1996:5) rappelle que, depuis le milieu du 19è siècle, les grammairiens distinguent plusieurs sortes de relations concessives, dont celle de concession logique, laquelle est généralement décrite comme l'expression d'une cause qui n'a pas été suivie

\footnotetext{
2 A la concession logique s'opposent, selon les distinctions de M.-A. Morel 1996, la concession rectificative et la concession argumentative. A propos de la concession argumentative, voir J.-Cl. Anscombre (1978, 1979, 1983, 1985 et 2002). Les structures concessives corrélatives telles que si ... que, quelque ... que sont décrites par C. Muller (1996:161-184) sous l'appellation de concessives extensionnelles.
} 
de l'effet attendu. Par exemple Sandfeld (1936:370 (\$ 223)) écrit que «les propositions concessives marquent un fait qui normalement devrait empêcher la réalisation d'un autre, mais qui n'a pas ou n'a pas en cet effet » et illustre cette affirmation des deux exemples suivants :

(5a) Il a cessé son travail, parce qu'il est malade.

(5b) Il n'a pas cessé son travail, bien qu'il soit malade.

Une manière plus détaillée de décrire ce qui se passe consiste à décrire les phrases à connecteur de concession comme étant l'expression de la négation externe d’une phrase présupposée à connecteur de cause.

\subsection{Notion de "négation externe "}

Il existe deux sortes de négation : la négation externe et la négation interne. Le fait est mis en évidence par Ducrot (1972:37-38 » et commenté, entre autres, par Nølke (1993, chapitre 4). La négation externe (que Ducrot 1972 appelle négation métalinguistique) est polémique: elle consiste à nier un énoncé présupposé. Par exemple, écrit Ducrot, l'énoncé ce mur n'est pas blanc « sera très rarement utilisé pour décrire un mur, et, de fait, il renseigne peu sur le mur considéré. La plupart du temps, on l'emploiera pour marquer qu'on s'oppose à une affirmation antérieure ce mur est blanc». Le cas de l'énoncé il n'y a pas un nuage dans le ciel est différent, ajoute Ducrot, puisqu'en disant cela « on entend la plupart du temps donner une description du ciel, dire comment il est ». Il s'agit alors de la négation interne (Ducrot l'appelle négation descriptive : elle sert, ditil, «à parler des choses, non des énoncés »).

La distinction entre les deux sortes de négations peut être exprimée en terme de portée de la négation:

(6) Négation externe : NEG-[ce mur est blanc] Ex. Ce mur n'est pas blanc.

Négation interne : [il non-existe un nuage dans le ciel]

Ex. Il n'y a pas un nuage dans le ciel.

Dans la négation externe, la négation a pour portée l'ensemble de la phrase qui correspond au présupposé. Dans la négation interne, elle a pour portée le seul prédicat de la phrase, ou le prédicat d'une éventuelle proposition subordonnée enchâssée. Dans les deux cas, on a appliqué le morphème de négation au prédicat de la phrase.

La notion de «négation externe » prend tout son sens dans le cadre de la théorie de la polyphonie (mise en scène de plusieurs énonciateurs, dont le locuteur (cf. entre autres Anscombre \& Ducrot 1988:174 \& sqq).

\subsection{La négation externe des énoncés à connecteur de cause}

Lorsque le connecteur de cause est un verbe linéarisant ses arguments dans l'ordre antécédent-conséquent, l'application de la négation externe à la phrase lui donne automatiquement un sens concessif (cf. Le Pesant 2005) :
(7a) NEG-le gel a causé des dégâts
Ex. Le gel n'a pas causé de dégâts.
NEG-la fatigue m'a obligé à m'arrêter
Ex. La fatigue ne m'a pas obligé à m'arrêter. 
Les exemples qui figurent dans (7a) sont synonymes de :

(7b) Malgré le gel, il n'y a pas eu de dégâts.

Malgré la fatigue, je n'ai pas été obligé de m'arrêter.

Les choses se passent autrement lorsqu'on cherche à appliquer la négation externe à des phrases dont le connecteur linéarise les arguments dans l'ordre conséquent-antécédent; c'est notamment le cas de la conjonction parce que. Reprenons l'exemple de la phrase (5a) et appliquons-lui le morphème de négation :

(5a) Il a cessé son travail, parce qu'il est malade.

(8) Il ñ'a pas cessé son travail, parce qu'il est malade.

La phrase (8) est grammaticale, mais elle n'a pas de sens concessif. Autrement dit, en utilisant simplement le morphème de négation, nous avons appliqué à cette phrase la négation interne.

La seule manière d'appliquer la négation externe à une phrase à connecteur conjonctif (ou prépositionnel) linéarisant les arguments dans l'ordre conséquent-antéédent est de la «transformer » en une phrase à connecteur concessif. Plus précisément, il faut : - appliquer le morphème de négation à l'argument conséquent;

- remplacer le connecteur de cause par un connecteur de concession.

Ainsi,

(9a) NEG externe de : conséquent Conjonction Causale antécédent

s'exprime au moyen de :

(9b) non-conséquent Conjonction Concessive antécédent

La négation externe de Il a cessé son travail, parce qu'il est malade est Il ña pas cessé son travail, bien qu'il soit malade.

\subsection{Deux types de relation de causalité : la causalité factuelle et la causalité opérant sur l'énonciation}

Une tradition inaugurée par O. Ducrot (1984) oppose la causalité opérant sur le dit (causalité factuelle) à la causalité opérant sur le dire, c'est-à-dire sur l'énonciation. Les énoncés inférentiels (déduction ou abduction) seront dès lors analysés à partir d'une primitive causale. Comparons parce que et puisque, qui sont les connecteurs les plus généraux de l'une et l'autre sorte. Le connecteur parce que a deux valeurs distinctes. Il exprime en général la causalité factuelle :

(10a) Pierre a le bras dans le plâtre parce qu'il a eu un accident.

Mais il peut aussi, avec de complexes restrictions d'emploi, exprimer la causalité opérant sur l'énonciation (cf. C. Rossari 2000:60-66). Ainsi l'énoncé (10b) a pour force illocutoire l'abduction :

(10b) Abduction: Pierre a eu un accident, parce qu'il a le bras dans le plâtre.

$=$ «Je dis que Pierre a eu un accident parce qu'il a le bras dans le plâtre» 
Quant à la conjonction puisque, elle ne peut pas exprimer la causalité factuelle. Elle exprime la causalité opérant sur l'énonciation, ce qui fait que l'énoncé dont elle est le prédicat a la force illocutoire soit de déduction, soit d'abduction:

(11a) Déduction : Pierre a le bras dans le plâtre, puisqu'il a eu un accident.

$=$ «Je dis que Pierre a le bras dans le plâtre parce qu'il a eu un accident»

(11b) Abduction : Pierre a eu un accident, puisqu'il a le bras dans le plâtre.

$=$ «Je dis que Pierre a eu un accident parce qu'il a le bras dans le plâtre »

\section{De la causalité à la condition}

Nous venons de «remonter» de la concession à la causalité (Section 2.3). Nous allons maintenant remonter encore davantage : de la causalité à la condition.

La relation entre la phrase à connecteur de cause (qu'il s'agisse de causalité factuelle ou de causalité opérant sur l'énonciation) et la phrase homologue à connecteur de condition peut être exprimée en termes de relation entre posé et présupposé. Soit la succession des trois énoncés suivants, qui constitue un syllogisme modus ponens:

(12a) Majeure : Elle n'a pas une figure d'enfant, si elle a 30 ans.

(12b) Conjonction de la majeure de la mineure:

(Elle n'a pas une figure d'enfant, si elle a 30 ans) ET (elle a 30 ans)

(12c) Conclusion : Elle n'a pas une figure d'enfant, parce qu'elle a 30 ans.

La phrase à connecteur de condition (12a) est une assertion, mais ses propositions constituantes ne sont pas assertées. L'énoncé (12b) a pour prédicat principal la conjonction de coordination et, dont l'argument de gauche est l'énoncé (12a) dans lequel la proposition elle a 30 ans n'est pas assertée ; quant à l'argument de droite, c'est de nouveau la proposition elle a 30 ans, assertée cette fois-ci.

La phrase à connecteur de cause (12c) est la conclusion (au sens logique) de l'ensemble : elle reprend l'assertion de elle a 30 ans déjà posée en (12b) et elle asserte la proposition elle n'a pas une figure d'enfant, ce que ne faisaient ni (12a), ni (12b).

Si on considère les choses en remontant de (12c) à (12a), nous pouvons dire que la phrase (12c) présuppose l'énoncé (12b) et au-delà (12a), puisque (12a) est déjà posé dans (12b).

\section{La contraposition des phrases à connecteur de condition, de causalité et de concession}

En logique élémentaire, on appelle loi de contraposition l'inférence immédiate suivante : «q $\boldsymbol{s i} p$ » est logiquement équivalent à «non- $p$ si non-q »

(13) On est bipède, si on est un homme $\equiv$ On n'est pas un homme, si on n'est pas bipède.

(12a) Elle n'a pas une figure d'enfant, si elle a 30 ans 三 Elle n'a pas 30 ans, si elle a une figure d'enfant. 


\subsection{La contraposition des phrases à connecteur de condition}

L'expression « être logiquement équivalent à » n'est pas synonyme de « être linguistiquement synonyme de ». Deux propositions sont logiquement équivalentes quand on peut remplacer l'une par l'autre et réciproquement salva veritate, c'est-à-dire sans toucher à la valeur de vérité, et ce indépendamment du contenu des arguments (l'équivalence logique est formelle). La synonymie de deux propositions implique certes la conservation de la valeur de vérité, mais elle prend en compte en plus le contenu linguistique des arguments. Revenons à la phrase (12a) et comparons-la à sa contraposée :
$(14 \mathrm{a})=(12 \mathrm{a})$
Elle n'a pas une figure d'enfant, si elle a 30 ans.
(14a') Contraposée de (14a)
Elle n'a pas 30 ans, si elle a une figure d'enfant.

Par la loi de contraposition, les phrases (14a) et (14a') sont logiquement équivalentes. Leur absence de synonymie peut être décrite en des termes de pragmatique: si on admet que c'est l'âge qui détermine le physique d'un individu et non pas l'inverse, il faut aussi admettre que (14)a est soit l'assertion de l'existence d'un fait, soit une déduction, alors que (14)a' est une abduction (cf. Section 2.4). Nous croyons possible de faire cette généralisation : étant donné deux propositions hypothétiques contraposées, l'une d'elles est l'assertion d'un fait ou une déduction, l'autre une abduction.

\subsection{La contraposition des phrases à connecteur de cause}

Revenons maintenant aux phrases à connecteur de cause qui, telles (14b) et (14c) présupposent une phrase à connecteur de condition (cf. Section 3) :

$\begin{array}{ll}(14 b)=(12 b) & \text { (Elle n'a pas une figure d'enfant, si elle a } 30 \text { ans) ET elle a } 30 \text { ans. } \\ (14 c)=(12 c) & \text { Elle n'a pas une figure d'enfant, parce qu'elle a } 30 \text { ans. }\end{array}$

Appliquons-leur la loi de contraposition là où c'est possible :

(14b') Contraposition de (14b) :

(Elle n'a pas 30 ans, si elle a une figure d'enfant) ET elle a une figure d'enfant.

$\left(14 c^{\prime}\right)$ Contraposition de (14c) :

Elle n'a pas 30 ans, parce qu'elle a une figure d'enfant.

Par abus de langage (car en logique la contraposition ne concerne que les énoncés à connecteur de condition), nous disons que les phrases à connecteur de cause qui présupposent une phrase conditionnelle contraposée sont elles-mêmes des «phrases contraposées à connecteur de cause ». Dans nos exemples, (14b) et (14b') d'une part, (14c) et (14c') d'autre part sont deux couples de phrases à connecteur de cause contraposées l'une à l'autre. Dans chaque couple, les phrases «contraposées à connecteur de cause» sont logiquement contradictoires (alors que les contraposées à connecteur de condition sont équivalentes). 


\subsection{La contraposition des phrases à connecteur de concession : retour à la propriété "porte-manteau "}

Nous allons maintenant appliquer la négation externe aux énoncés (14c) et $\left(14 c^{\prime}\right)$, afin d'obtenir des phrases à connecteur de concession. Selon la procédure décrite dans la Section 2.3, nous devons appliquer le morphème de négation à l'argument conséquent et remplacer le connecteur de cause par un connecteur de concession. Nous obtenons les phrases (15) et (15') :

(15) Négation externe de (14c): Elle a une figure d'enfant, bien qu'elle ait 30 ans.

(15') Négation externe de (14c'): Elle a 30 ans, bien qu'elle ait une figure d'enfant.

Par le même abus de langage que celui qui nous a fait parler de "phrases contraposées à connecteur de cause ", nous disons que les phrases (14c) et (15) d'une part, et les phrases $\left(14 c^{\prime}\right)$ et $\left(15^{\prime}\right)$ d'autre part sont des "phrases contraposées à connecteur de concession ». Deux phrases à connecteur de concession contraposées l'une à l'autre sont logiquement contradictoires : leur relation n'est pas d'équivalence, mais de disjonction exclusive.

Les deux membres de chaque couple de phrases contraposées à connecteur de concession se caractérisent formellement par l'ordre inverse de leurs arguments : on retrouve là le phénomène de permutation d'arguments évoqué dans l'Introduction et dans la Section 1.

\section{Récapitulation : une classe d'équivalence de phrases à connecteur de condition, de cause et de concession}

Nous sommes en présence du système de phrases suivant:

Si $\mathrm{p}=$ « elle a 30 ans » et $\mathrm{q}=$ «elle n'a pas une figure d'enfant », alors :
(14a) majeure
EQUIVAUT A
(14a') contraposée de (14a)
$\mathrm{q} s i \mathrm{p}$
non-p $s i$ non-q
Elle n'a pas une figure d'enfant,
Elle n'a pas 30 ans,
si elle a 30 ans.
si elle a une figure d'enfant.
(14b) conjonction majeure/mineure OU BIEN
(q si p) et $\mathrm{p}$
Elle n'a pas une figure d'enfant,
$\left(14 b^{\prime}\right)$ « contraposée » de (14b)
(non-p si non-q) et non-p
Elle n'a pas 30 ans, si elle a
si elle a 30 ans. Or elle a 30 ans.
une figure d'enfant.
Or elle a une figure d'enfant.
(14c) conclusion de (14b)
OU BIEN (14c') «contraposée » de (14c)
q parce que $\mathrm{p}$
Elle n'a pas une figure d'enfant, non-p parce que non-q
Elle n'a pas 30 ans,
parce qu'elle a 30 ans.
parce que (= puisque) elle a une
figure d'enfant. 
(15)

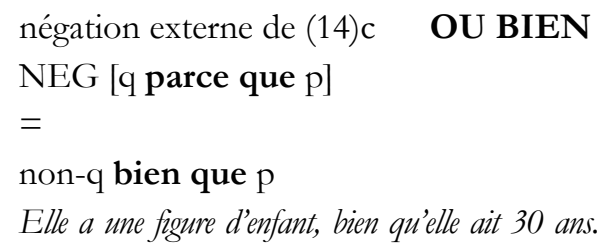

$\left(15^{\prime}\right)$ négation externe de $\left(14 \mathrm{c}^{\prime}\right)$

NEG [non-p parce que q] $=$

$\mathrm{p}$ bien que non-q

Elle a 30 ans, bien qu'elle ait une figure d'enfant

Ce système met en jeu trois types de relations :

i) une série d'inférences immédiates formant le syllogisme modus ponens (14a-c)

ii) une série d'applications de la loi de contraposition (14a/a'), (14b/b'), (14c/c')

iii) deux applications de la négation externe : $(14 \mathrm{c}) /(15)$ et $\left(14 \mathrm{c}^{\prime}\right) /\left(15^{\prime}\right)$.

C'est une classe d'équivalences syntaxiques en ce sens que c'est un ensemble de phrases reliées par une série de constantes sémantiques. Mais ce n'est pas une classe d'équivalences transformationnelles (cf. Section 7).

\section{Le cas des connecteurs adverbiaux de concession logique}

Nous évoquons pour finir les adverbes de concession pourtant et cependant. Reprenons deux exemples de Morel (1996) illustrant leur propriété « porte-manteau » :

(16) Notre voiture a 100.000 bornes, elle a pourtant (cependant) très bien roulé.

(17) Notre voiture a très bien roulé, elle a pourtant (cependant) 100.000 bornes.

Il a été souvent noté (avec des arguments fondés entre autres sur l'étymologie) que les adverbes connecteurs ont une valeur anaphorique: alors comme substitut de «au moment où cela se passait», ensuite comme substitut de «à la suite de cela », c'est pourquoi comme substitut de "c'est pour cela que », donc comme substitut de «d'où », «de là vient que» etc. Il y a tout lieu de penser qu'il en va de même pour les connecteurs de concession logique pourtant, cependant, toutefois, néanmoins, pour autant, malgré tout.... Ils sont tous fondamentalement, mais avec des différences dans les conditions d'emploi, des substituts de «malgré cela»: ils cumulent la nature de connecteur concessif et celle d'anaphore. Voici une illustration de cette analyse :

(16a) [Notre voiture a 100.000 bornes $]_{i}$; elle a pourtant $\left[=\right.$ malgré $\left.(c e l a)_{i}\right]$ très bien roulé.

L'énoncé (16) est composés de deux phrases. Contrairement aux apparences, l'adverbe concessif pourtant ne sert pas à connecter les deux phrases : la relation concessive n'est présente qu'au sein de la deuxième phrase. C'est ce qu'illustre l'analyse (16a).

Voici maintenant l'analyse de l'énoncé (17), contraposition de (16) :

(17a) $[\text { Notre voiture a très bien roulé }]_{i}$; elle a pourtant $\left[=\right.$ malgré $\left.(\text { cela })_{i}\right] 100.000$ bornes.

Ces analyses impliquent que :

(16) Notre voiture a 100.000 bornes, elle a pourtant (cependant) très bien roulé. $=$ Notre voiture a très bien roulé bien que (quoique) elle ait 100.000 bornes. 
et que

(17) Notre voiture a très bien roulé, elle a pourtant (cependant) 100.000 bornes.

$=$ Notre voiture a 100.000 bornes bien que (quoique) elle ait très bien roulé.

Le caractère paradoxal de ces relations de synonymie est dû au fait que le caractère anaphorique des connecteurs concessifs adverbiaux n'est pas évident.

\section{Classes d'équivalences transformationnelles et classes d'équivalences non transformationnelles}

Nous nous sommes abondamment référé à la loi de contraposition: « q si p » $三$ «non-p si non-q" (Si l'eau dépasse $100^{\circ}$, elle bout $\equiv$ Si l'eau ne bout pas, elle ne dépasse pas $100^{\circ}$. Nous nous sommes également référé à une autre inférence immédiate de la logique élémentaire, la loi de double négation, quand nous avons écrit, par exemple à propos de la phrase (15'), que «avoir 30 ans» est la négation de «ne pas avoir 30 ans». D'autre part, nous avons ordonné une série de structures de phrases en syllogisme modus ponens. Enfin, l'analyse des phrases concessives en termes de négation externe d'un énoncé à connecteur causatif met en jeu un phénomène de changement de portée de la négation.

A-t-on le droit, en syntaxe et en sémantique des langues naturelles, de créer de la sorte des classes d'équivalences fondées sur telle ou telle tautologie de la logique élémentaire? Ne doit-on pas se borner à étudier les variantes syntaxiques qui concernent le nombre des arguments, leur ordre, leurs «effacements", leurs «mouvements», leurs pronominalisations, etc. ? Autrement dit, ne doit-on pas se limiter aux équivalences transformationnelles? Nous répondons à ces questions par la négative. En effet, il existe un trop grand nombre de régularités syntacticosémantiques non transformationnelles pour qu'on puisse se permettre d'en faire abstraction dans l'analyse linguistique.

Prenons l'exemple des régularités paraphrastiques de conversion. Certes, un certain nombre d'entre elles sont des transformations. Par exemple, on peut parler de transformation de conversion (ou réciprocité) quand la permutation des arguments, pour un même prédicat, conserve le sens. C'est ce qui se passe avec des verbes comme se marier, se brouiller et se battre:

(18) Pierre s'est (marié, brouillé, battre) avec Marie.

= Marie s'est (mariée, bronillée, battue) avec Pierre = Ils se sont (mariés, brouillés, battus).

On peut également parler de transformation de conversion quand, pour un même prédicat nominal, elle se fait au moyen d'un verbe support spécialisé (cf. G. Gross (1989)) :

(19) Pierre a donné un conseil à Marie = Marie a reçu un conseil de Pierre.

Il est en revanche impossible, comme l'indique Z. Harris (1990:13), d'attribuer un caractère transformationnel aux paraphrases de conversion qui mettent en relation des prédicats différents, telles : 
(20) Pierre a (prêté, vendu) un livre à Marie $=$ Marie a (emprunté, acheté) un livre à Pierre.

(21) Pierre a informé Marie qu'il veut l'épouser = Marie a appris de Pierre qu'il veut l'épouser.

Dans des cas de ce genre, la synonymie n'est pas corrélée à une transformation. Il en va de même pour le phénomène de conversion qui met en jeu les relations cause/ conséquence et conséquence/ cause, comme dans :

(22) Je suis parti, parce que j'étais fatigué = J'étais fatigué, si bien que je suis parti.

Se priver d'étudier les régularités non transformationnelles reviendrait à se condamner à passer à côté d'une énorme partie du système de la langue. Aussi croyons-nous qu'en syntaxe et en sémantique, il faut, à côté des variantes paraphrastiques transformationnelles, prendre en compte, comme nous venons de le faire, les régularités paraphrastiques non transformationnelles. 
Denis Le Pesant

\section{RÉFÉRENCES BIBLIOGRAPHIQUES}

Anscombre, J.-C., 1983, « Pour autant, pourtant (et comment): à petites causes, grands effets ». Cabier de Linguistique Française 5 : Genève 37-85.

ANSCOMBRE, J.-C., 1985, «Grammaire traditionnelle et grammaire argumentative de la concession ». Revue Internationale de Philosophie vol. 39, n¹55 : 333-350.

Anscombre, J.-C., 2002, «Mais pourtant dans la contre-argumentation directe » in Danielle Leeman (éd.), LINX, Les connecteurs, nº $46: 155-131$.

Anscombre, J.-C. \& Ducrot, O., 1978, «Lois logiques et lois argumentatives 1 », Le Français Moderne, vol. 46 n². Paris, d'Artrey : 347-357.

Anscombre, J.-C. \& Ducrot, O., 1979, «Lois logiques et lois argumentatives 2 ». Le Français Moderne, vol. 47 n¹. Paris, Hachette : 35-61.

Anscombre, J.-C. \& Ducrot, O., 1988, L'argumentation dans la langue. Liège, Mardaga.

Ducrot, O., 1972, Dire et ne pas dire. Paris, Hermann.

Ducrot, O., 1984, Le dire et le dit. Paris, Editions de Minuit.

Gross, G., 1989, Les constructions converses en français. Genève, Paris, Librairie Droz.

HARris, Z., 1976, Notes du cours de syntaxe. Paris, Le Seuil.

HARris, Z., 1990, «La genèse de l'analyse des transformations et de la métalangue ». Langages 99. Les grammaire de Harris et leurs questions (A. Daladier éd.). Paris, Larousse : 9-20.

LE Pesant, D., 2005, "Causalité et concession », in Choi-Jonin, Bras, Dagnac \& Rouquier (éds), Questions de classification en linguistique. Mélanges offerts au Professeur Christian Molinier. Bern : Peter Lang. : 195-209.

MOREL, M-A., 1996, La concession en français. Gap, Ophrys.

MulLER, C., 1996, La subordination en français. Paris, Armand Colin.

NøLKE, H., 1993, Le regard du locuteur. Paris, Editions Kimé.

Rossari, C., 2000, Connecteurs et relations de discours. Nancy, Presses Universitaires.

SANDFELD, Kr., 1936, Syntaxe du français contemporain. Les propositions subordonnées. Genève, Droz. 